\title{
Application of Artificial Intelligence-based Dual- modality Analysis Combining Fundus Photography and Optical Coherence Tomography in Diabetic Retinopathy Screening in a Community Hospital
}

Rui Liu

Shanghai Jing'an District Shibei Hospital

Qingchen Li

Fudan University

Feiping Xu

Shanghai Jing'an District Shibei Hospital

Shasha Wang

Shanghai Jing'an District Shibei Hospital

Jie He

Shanghai Jing'an District Shibei Hospital

Yiting Cao

Shanghai Jing'an District Shibei Hospital

Fei Shi

Soochow University

Xinjian Chen

Soochow University

Jili Chen ( $\square$ corneachen@163.com )

Shanghai Jing'an District Shibei Hospital

\section{Research Article}

Keywords: Artificial intelligence, Deep learning,Diabetic retinopathy, Optical coherence tomography

Posted Date: December 17th, 2021

DOI: https://doi.org/10.21203/rs.3.rs-1158235/v1

License: (c) (1) This work is licensed under a Creative Commons Attribution 4.0 International License.

Read Full License 


\section{Abstract}

Background To assess the feasibility and application value of artificial intelligence (AI)-based screening for diabetic retinopathy (DR) by combining fundus photos and optical coherence tomography (OCT) images in a community hospital.

Methods Fundus photos and OCT images were taken for 600 diabetic patients in a community hospital. Ophthalmologists and Al identified these fundus photos according to international DR standards. OCT images were used to detect concomitant macular oedema (ME). The criteria for referral were DR grades 24 and/or the presence of ME. The sensitivity and specificity of Al grading were evaluated. The number of referable DR cases confirmed by ophthalmologists and Al was calculated.

Results DR was detected in 81 (13.5\%) participants by ophthalmologists and in 94 (15.6\%) by Al, and 45 (7.5\%) and 53 (8.8\%) participants were diagnosed with referable DR by ophthalmologists and by $\mathrm{Al}$, respectively. The sensitivity, specificity and area under the curve (AUC) of Al for detecting DR were $91.67 \%, 96.92 \%$ and 0.944 , respectively. For detecting referable DR, the sensitivity, specificity and AUC of Al were $97.78 \%, 98.38 \%$ and 0.981 , respectively. ME was detected from OCT images in 49 (8.2\%) participants by ophthalmologists and in 57 (9.5\%) by Al, and the sensitivity, specificity and AUC of Al were $91.30 \%, 97.46 \%$ and 0.944 , respectively. When combining fundus photos and OCT images, the number of referrals identified by ophthalmologists increased from 45 to 75 and from 53 to 85 by Al.

Conclusion Al-based DR screening has high sensitivity and specificity and may feasibly improve the referral rate of community DR.

\section{Background}

Diabetic retinopathy (DR), one of the most common complications of diabetes mellitus, is an eye disease known to cause moderate to severe visual loss and is the leading cause of blindness in working-age people suffering from long-standing diabetes[1-3]. Very often, the disease does not show overt symptoms until it reaches an advanced stage. However, a regular follow-up can allow early detection and treatment of vision-threatening retinopathy, which enables the prevention of up to $98 \%$ of visual loss due to DR[4, 5]. Given that most vision loss from DR is avoidable through early detection and effective treatment strategies[6, 7], many national and international societies have long endorsed screening for DR[8], which is most commonly in the form of point-of-care ophthalmoscopy by trained ophthalmologists or retinal photography with either local interpretation or telemedicine-based screening programs with centralized grading[9].

In recent years, with the improvement of computer processing speed, artificial intelligence (Al) programs have better assisted in the diagnosis and management of clinical diseases, especially in the field of ophthalmology. The application of this technology in ophthalmology is currently focused mainly on diseases with a high incidence, such as DR and age-related macular degeneration (ARMD)[10]. Several current studies have proposed Al screening algorithms for DR, which show high accuracy, sensitivity and 
specificity[11-19]. We recently applied Al-based DR screening in community hospitals and achieved high sensitivity and specificity in detecting DR and referable diabetic retinopathy (RDR)[20]. However, in the process of screening, we also found some deficiencies, especially in the identification of diabetic macular oedema (DME). As a diagnosis of DME requires identification of macular thickening, screening for DME using non-steresoscopic fundus photographs is likely to cause errors. Therefore, if optical coherence tomography (OCT), as the primary tool for macular disease detection, can be introduced as a screening test in addition to fundus photography, the sensitivity to detect fundus diseases will increase further. To the best of our knowledge, the simultaneous use of fundus photography and OCT for the screening of DR has not been reported before. Herein, we applied deep learning-based Al grading of DR based on both fundus photography and OCT to community hospital clinics. This study aims to assess the accuracy of Al-based screening for DR by fundus photos combined with OCT images and to explore the feasibility and application value of this dual-modality for DR screening in a community hospital.

\section{Results}

Six hundred diabetic patients participated in the DR screening, including 324 men and 276 women, and for each person, a random eye was taken for testing. The average age of the participants was $67.26 \pm$ 7.02 years. For fundus photography, 1200 retinal images were obtained and graded in this study. According to the International Clinical Diabetic Retinopathy (ICDR) classification scale, 78 participants with DR were detected by both ophthalmologists and Al. Ophthalmologists detected DR in 81 (13.5\%) participants, while Al detected DR in 94 (15.6\%) participants. Fig. 3 shows an OCT-Fundus-Al diagnostic result compared with those of single modality-based diagnosis systems, which indicates the superiority of the multi-modality-based retinal disease diagnostic system. Most of the participants' fundus photographs revealed no DR. RDR was diagnosed in 45 (7.5\%) participants based on manual grading and in $53(8.8 \%)$ participants using $\mathrm{Al}$ (Fig. 4a). For DR detection, the sensitivity and specificity achieved by Al were $91.67 \%$ (95\% Cl77.5-98.2) and 96.92\% (95\% Cl 95.0-98.2), respectively. For RDR detection, the sensitivity and specificity achieved by Al were $97.78 \%(95 \% \mathrm{Cl} 88.2-99.9)$ and $98.38 \%(95 \% \mathrm{Cl} 96.9-$ 99.3), respectively. The area under the curve (AUC) was 0.944 (95\% Cl 0.922-0.962) when testing the ability of Al to detect DR; for the detection of RDR, the AUC was 0.981 (95\% Cl $0.966-0.990)$.

For OCT images, 43 participants with ME were detected by both ophthalmologists and Al, 20 of whom were considered to have DME. ME was detected by ophthalmologists in $49(8.2 \%)$ participants and in 57 (9.5\%) participants by Al(Fig. 4b). For ME detection, the sensitivity, specificity and AUC of Al were $91.30 \%$ (95\% Cl 72.0-98.9), 97.46\% (95\% Cl 95.8-98.6) and 0.944 (95\% Cl 0.922-0.962), respectively. A matched diagnosis of RDR between ophthalmologists and Al grading was observed in 44 participants by fundus photography. However, when combined with OCT, the number of referrals given by ophthalmologists increased from 45 to 75 , where the increase included 7 for DME and 23 for other reasons. For Al, the number of referrals increased from 53 to 85 . The number of referrals jointly identified by ophthalmologists and Al also increased to 71, as shown in Fig. 5 and Table 1. 
Sensitivity, specificity and AUC of Al for detection of different degrees of DR and ME with ophthalmologist grading as reference standard.

\begin{tabular}{|c|c|c|c|c|c|c|c|}
\hline Stage & $\begin{array}{l}\text { Sensitive, } \\
\%\end{array}$ & $\triangle 95 \% \mathrm{Cl} \nabla$ & $\begin{array}{l}\text { Specificity, } \\
\%\end{array}$ & $\triangle 95 \% \mathrm{Cl} \otimes$ & AUC & $\triangle 95 \% \mathrm{Cl} \nabla$ & P Value \\
\hline $\begin{array}{l}\text { Any } \\
\text { DR }\end{array}$ & 91.67 & $\begin{array}{l}77.5- \\
98.2\end{array}$ & 96.62 & $\begin{array}{l}95.0- \\
98.2\end{array}$ & 0.944 & $\begin{array}{l}0.922- \\
0.962\end{array}$ & $\otimes 0.0001$ \\
\hline RDR & 97.78 & $\begin{array}{l}88.2- \\
99.9\end{array}$ & 98.38 & $\begin{array}{l}96.9- \\
99.3\end{array}$ & 0.981 & $\begin{array}{l}0.966- \\
0.990\end{array}$ & $\otimes 0.0001$ \\
\hline ME & 91.30 & $\begin{array}{l}72.0- \\
98.9\end{array}$ & 97.46 & $\begin{array}{l}95.8- \\
98.6\end{array}$ & 0.944 & $\begin{array}{l}0.922- \\
0.962\end{array}$ & $\llbracket 0.0001$ \\
\hline
\end{tabular}

\section{Discussion}

The Al system used in this study can achieve relatively good results after a short period of training and learning using a small batch of data. In the past five years, the sensitivity and specificity of the Alassisted DR screening system based on colour fundus photographs reported by various working groups around the world were $87 \%-99 \%$ and $91.4 \%-99.0 \%$, respectively, and the AUC was $0.989-0.991$ [11-19]. Our results showed that the sensitivity of DR screening using Al was $91.67 \%$, the specificity was $96.92 \%$ and the AUC was 0.944 , which are similarto those in previous studies and may therefore meet the needs of clinical screening. Moreover, the sensitivity of detecting RDR in our study was $97.78 \%$, and the AUC was 0.981[17], which are close to the results of the former study, where the authors demonstrated the performance of a deep learning enhancement algorithm used for automatic RDR detection. The sensitivity of their algorithm was $96.8 \%$, and the AUC was 0.980 . Moreover, the specificity of our results was much higher, reaching $98.38 \%$ compared with $87 \%$. Additionally, the sensitivity for RDR detection in our study was much higher than that for DR detection, indicating that more advanced disease corresponds to more accurate identification by our system.

DME is the most common cause of visual loss in those with diabetic retinopathy and is increasing in prevalence globally[21-23].The prevalence of DME in patients with diabetic retinopathy is $2.7 \%-11 \%$ and depends on the type of diabetes and the duration of the disease, but for both types 1 and 2, after 25years of duration, the prevalence is approximately $30 \%[21]$. In DME, the macula is thickened due to increased extracellular fluid derived from hyperpermeable retinal capillaries, affecting detailed central vision. Notably, DME can occur at any stage of DR, whether non-proliferative diabetic retinopathy (NPDR) or proliferative diabetic retinopathy (PDR)[24].However, before the use of OCT, the detection of macular oedema in clinical studies was performed with 2-dimensional (2-D) non-steresoscopic digital fundus photography. Without stereopsis, monocular fundus photography studies identified DME using surrogate markers of thickening, such as lipids near the foveal centre, macular focal/grid laser scars, or localized colour changes in the macula[25-27].Therefore, affected by pupil size and refractive media, screening for DME using non-steresoscopic fundus photographs is likely to have a very high false-positive rate (e.g., $>86 \%$ in Hong Kong [28] and $>79 \%$ in the UK [29]). Recent studies have focused on demonstrating that 
deep learning algorithms can be trained using OCT images to detect DME and other retinal diseases.Kermany et al. [30]first applied deep learning and transfer learning techniques in the detection of ARMD and DME from 2-D OCT images. Their models achieved high performance (sensitivity $\geq 96 \%$ and specificity $\geq 94 \%$ ). The latest study is from Wang et al. [31]who applied a deep learning model with an adapted feature pyramid network to detect 15 categories of retinal pathologies from OCT images as common signs of various retinal diseases. Their results also reached a high level with a sensitivity $\geq 94 \%$ and specificity $\geq 98 \%$.

In our study, we used OCT not only to identify macular oedema but also to accurately identify ARMD, subretinal fluid, and other diseases. However, due to the small sample size of these diseases, we did not perform separate statistics for these diseases. Our statistical analysis showed that the sensitivity of ME screening using AI was $91.30 \%$, the specificity was $97.46 \%$, and the AUC was 0.944 , which are slightly lower than those of previously reported cases, possibly because our study was a real-world study conducted in community hospitals. The subjects were generally older, and some subjects had cataracts and other diseases at the same time, which may affect the quality of some pictures. When fundus photography was combined with OCT, the number of referrals increased from 45to 75, including 7 for DME and 23 for other reasons. Although we detected only 7 patients with DME in this screening, retinal thickness abnormalities caused by other causes should not be ignored, and reliable referral can also allow these patients to receive treatment as soon as possible.Thus, OCT appears to provide ophthalmologists with a critical reference for clinical diagnosis. It can reduce not only the rate of missed diagnosis but also inappropriate referral caused by false-positives.

The advantage of this study is that our algorithm can process and analyse fundus photography and OCT at the same time. Our study and previous studies have shown that Al-based DR screening for outpatients seems to be feasible. After the examination, Al will issue the report on-site without waiting. The doctors in the community hospital can address the report according to the condition of the patient and select the appropriate patients for referral to an ophthalmologist at a superior hospital. During the examination, none of the patients had mydriasis, which was more easily accepted.

Currently, most Al-assisted screening for DR has been carried out worldwide in the form of simple fundus photography. Based on this practice, we applied innovations and proposed for the first time that OCT can improve the screening rate and accuracy, detect patients with early DME, and increase the number of effective referrals. In the future, in economically developed countries as well as some developed areas in developing countries, OCT can also be used as a screening tool in a dual-modality combination that may lead to the identification of more patients with early DR.

Although our research has achieved good results, several limitations of this study must be considered. First, the datasets used in this study were collected from only one community hospital, and the subjects were generally elderly and could not cover all age groups. Second, the sample size was relatively small, resulting in an uneven distribution of patients with different grades of retinopathy. 


\section{Conclusion}

In this study, both Al-assisted DR screening systems based on colour fundus photographs and Al-assisted ME screening systems based on OCT have high sensitivity and specificity. This system can be feasibly implemented in the outpatient clinic of a community hospital, and more patients requiring referral can be identified to improve the referral rate of community DR.

\section{Methods}

\section{Participants}

This study was performed following the tenets of the Declaration of Helsinki. Diabetes patients who attended Pengpu Town Community Hospital of Jing'an district, Shanghai, were invited to participate in this study. All participants had no systemic diseases that could affect the retina except for diabetes. All subjects were 18 years of age or older, and written informed consent was obtained from each subject. This study was approved by the ethical committee of Shibei Hospital, Jing'an District, Shanghai (ChiCTR1900024528).

\section{Datasets}

All the images in this study were acquired using the Topcon 3-dimensional OCT-1 Maestro (Topcon, Tokyo, Japan), which could acquire both colour fundus and OCT images. For fundus photography, $45^{\circ}$ colour retinal photographs were taken for each eye. Retinal images with two fields, macula-centred and disc-centred, were captured according to the EURODIAB protocol [32]. For OCT, 50,000 axial scans were captured per second, producing a $20-\mu \mathrm{m}$ lateral and $6-\mu \mathrm{m}$ axial resolution. All the OCT images had a field of view of $6 \mathrm{~mm} \times 6 \mathrm{~mm}$. Al equipment was installed and used in the community hospital. Images of each participant were immediately analysed by Al and transmitted to two ophthalmologists simultaneously. Patient information was anonymized and unconnected to OCT images before transfer to the investigators. All the included images could be accurately diagnosed by retinal specialists, and images that were unclear because of hazy media, such as serious cataracts, fixation failure during image capture, severe motion or shadow artefacts, and other reasons, were excluded.

\section{Human grading}

All fundus photographs and OCT images were graded independently by two ophthalmologists (retina specialists, Kappa $(\mathrm{K})=0.844,0.864)$ who were masked to each other and Al device outputs. When the results between the two retina specialists were inconsistent, a third retina specialist made a final decision. All three experts had more than ten years of experience. The grading of retinopathy was evaluated according to the ICDR severity scale[8]. OCT images of those patients were obtained by Al and 
ophthalmologists to identify concomitant ME. The criteria for referralwere DR grades 2 (moderate NPDR), 3 (severe NPDR), and 4 (PDR)and/or the presence of ME [33].

\section{Automated grading}

The Al device performed automated analysis and identified signs from both retinal photographs and OCT images with Al software (Bigvision Inc., Suzhou, China).Then,a DR screening report including referral recommendations was generated and delivered to the participant immediately. This multimodality retinal disease diagnosis system applied deep learning algorithms to fundus images and OCT scans to achieve disease classification and detection and integrated the results to generate a comprehensive screening report with referral recommendations. The specific deep learning framework used was ResNet 101 for DR stage classification on fundus images and Faster-RCNN for retinal abnormality detection on OCT scans [34].

For fundus photography, the classification network of our proposed diagnostic system was based on ResNet 101, which was initialized by the pre-trained model trained on the ImageNet dataset. Since DR lesions such as micro aneurysms appeared as a small region, down sampling of the input fundus image might cause the lesion regions to disappear.Therefore, a high-resolution input size of $1024 \times 1024$ was used, which strongly ensured that the small lesion regions were not eliminated by down-sampling preprocessing. As described in our previous work, we collected a large-scale dataset for the training of the DR classification network [35], which contained more than 50,000 labelled fundus images. During the training, an uneven training batch data selection mechanism was adopted to overcome the problem of class imbalance. Fig. 1 showsthat the Al software yields DR stage classification results based on the values of the output vector from the heatmap.

For OCT, the detection network was built based on Faster R-CNN and combined with the mutual information between retinal layers for accurate retinal abnormality localization and classification[35]. The training dataset contains 25,000 OCT B-scans from more than 2,000 patients, involving 16 types of common retinal abnormalities. The ResNet 101 architecture was used as the backbone model for feature extraction from input OCT B-scans, and the output predicted bounding boxes of lesions and class labels were further filtered based on 3D spatial context information from the volumetric scan.Fig. 2 shows retinal abnormalities detection results from OCT B-scans by our OCT-Al diagnostic system.

Our classification and detection networks were implemented by the PyTorch framework and trained on a deep learning server with eightGeForce GTX 1080ti graphical processing units. The classification network was initialized by the pre-trained model trained on the ImageNet dataset. The Adam optimizer was used for training with 10,000 epochs, and the initial learning rate was 0.0001 . For the detection networks, the Adam optimizer was used for training with 10,000 epochs, and the initial learning rate was 0.0001 . To improve the generalization abilities of our networks, we augmented the training data by random flipping, random cropping, and random noise addition. 


\section{Statistical analysis}

Statistical analysis of the data was performed using the SPSSStatistics 26.0 for Windows (SPSS Inc., Chicago,IL, USA). The consistency between two ophthalmologists was evaluated using the Kappa coefficient, and the sensitivity and specificity of Al automatic grading were calculated regarding the results from ophthalmologists as the gold standard. The number of referrals confirmed by Al and the ophthalmologists was calculated and compared.

\section{Abbreviations}

Al: artificial intelligence

DR: diabetic retinopathy

OCT:optical coherence tomography

ME: macular oedema

AUC: area under the curve

ARMD: age-related macular degeneration

RDR: referable diabetic retinopathy

DME: diabetic macular oedema

2-D: 2-dimensional

ICDR: International Clinical Diabetic Retinopathy

\section{Declarations}

\section{Ethics approval and consent to participate}

All study procedures were performed in accordance with the tenets of the Declaration of Helsinki, and the Study was approved by the Ethics Committee of Shanghai Jing'an District Shibei Hospital. Written informed consent was obtained from all the study participants.

\section{Consent to publish}

Not applicable.

\section{Availability of data and materials}


The datasets used and/or analysed during the current study available from the corresponding author on reasonable request.

\section{Competing interests}

The authors have no proprietary or commercial interest in any materials discussed in this article.

\section{Funding}

This work was supported by the Advanced and Appropriate Technology Promotion Project of Shanghai Health Commission (2019SY012), National Key R\&D Program of China under Grant (2018YFA0701700), Shanghai Medical Key Special Construction Project (ZK2019B27), Project of Shanghai Municipal Commission of Health and Family Planning (20204Y0037), Project of Shanghai Jing'an District Municipal Commission of Health and Family Planning (2018MS12, 2020QN05), and Shanghai Jing'an District Shibei Hospital Research Project Grant (2020SBYMZB01). These funding sources had no role in the design of this study and will not have any role during its execution, analyses, interpretation of the data, or decision to submit results.

\section{Authors' contributions}

Rui Liu,Qingchen Li andFeiping Xu drafted and revised the manuscript.

Shasha Wang,Jie He, and Yiting Caocollected the data and performed the statistical analysis.

Fei Shi and Xinjian Chen constructed algorithm and optimized software.

Jili Chenconceived the studyand approved the final manuscript.

All authors have read and approved the manuscript.

\section{Acknowledgements}

None.

\section{References}

1. Teo ZL, Tham YC, Yu M, Cheng CY, Wong TY, Sabanayagam C: Do we have enough ophthalmologists to manage vision-threatening diabetic retinopathy? A global perspective. Eye (Lond)2020, 34(7):1255-1261. 
2. Yau JW, Rogers SL, Kawasaki R, Lamoureux EL, Kowalski JW, Bek T, Chen SJ, Dekker JM, Fletcher A, Grauslund Jet al: Global prevalence and major risk factors of diabetic retinopathy. Diabetes Care2012, 35(3):556-564.

3. Leasher JL, Bourne RR, Flaxman SR, Jonas JB, Keeffe J, Naidoo K, Pesudovs K, Price H, White RA, Wong TYet al: Global Estimates on the Number of People Blind or Visually Impaired by Diabetic Retinopathy: A Meta-analysis From 1990 to 2010. Diabetes Care2016, 39(9):1643-1649.

4. Doi K: Diagnostic imaging over the last $\mathbf{5 0}$ years: research and development in medical imaging science and technology. Phys Med Biol2006, 51(13):R5-27.

5. Solomon SD, Chew E, Duh EJ, Sobrin L, Sun JK, VanderBeek BL, Wykoff CC, Gardner TW: Diabetic Retinopathy: A Position Statement by the American Diabetes Association. Diabetes Care2017, 40(3):412-418.

6. Cheung N, Mitchell P, Wong TY: Diabetic retinopathy. Lancet2010, 376(9735):124-136.

7. Wong TY, Cheung CM, Larsen M, Sharma S, Simo R: Diabetic retinopathy. Nat Rev Dis Primers2016, 2:16012.

8. Wilkinson CP, Ferris FL, 3rd, Klein RE, Lee PP, Agardh CD, Davis M, Dills D, Kampik A, Pararajasegaram R, Verdaguer JTet al: Proposed international clinical diabetic retinopathy and diabetic macular edema disease severity scales. Ophthalmology2003, 110(9):1677-1682.

9. Goh JK, Cheung CY, Sim SS, Tan PC, Tan GS, Wong TY: Retinal Imaging Techniques for Diabetic Retinopathy Screening. J Diabetes Sci Techno/2016, 10(2):282-294.

10. Padhy SK, Takkar B, Chawla R, Kumar A: Artificial intelligence in diabetic retinopathy: A natural step to the future. Indian J Ophthalmol2019, 67(7):1004-1009.

11. Ramachandran N, Hong SC, Sime MJ, Wilson GA: Diabetic retinopathy screening using deep neural network. Clin Exp Ophthalmol2018, 46(4):412-416.

12. Gulshan V, Peng L, Coram M, Stumpe MC, Wu D, Narayanaswamy A, Venugopalan S, Widner K, Madams T, Cuadros Jet al: Development and Validation of a Deep Learning Algorithm for Detection of Diabetic Retinopathy in Retinal Fundus Photographs. JAMA2016, 316(22):2402-2410.

13. Gargeya R, Leng T: Automated Identification of Diabetic Retinopathy Using Deep Learning. Ophthalmology2017, 124(7):962-969.

14. Hemanth DJ, Anitha J, Son LH, Mittal M: Diabetic Retinopathy Diagnosis from Retinal Images Using Modified Hopfield Neural Network. J Med Syst2018, 42(12):247.

15. Abramoff MD, Lavin PT, Birch M, Shah N, Folk JC: Pivotal trial of an autonomous Al-based diagnostic system for detection of diabetic retinopathy in primary care offices. NPJ Digit Med2018, 1:39.

16. Ting DSW, Cheung CY, Lim G, Tan GSW, Quang ND, Gan A, Hamzah H, Garcia-Franco R, San Yeo IY, Lee SY et al: Development and Validation of a Deep Learning System for Diabetic Retinopathy and Related Eye Diseases Using Retinal Images From Multiethnic Populations With Diabetes. JAMA2017, 318(22):2211-2223. 
17. Abramoff MD, Lou Y, Erginay A, Clarida W, Amelon R, Folk JC, Niemeijer M: Improved Automated Detection of Diabetic Retinopathy on a Publicly Available Dataset Through Integration of Deep Learning. Invest Ophthalmol Vis Sci2016, 57(13):5200-5206.

18. Li Z, Keel S, Liu C, He Y, Meng W, Scheetz J, Lee PY, Shaw J, Ting D, Wong TYet al: An Automated Grading System for Detection of Vision-Threatening Referable Diabetic Retinopathy on the Basis of Color Fundus Photographs. Diabetes Care2018, 41(12):2509-2516.

19. Kanagasingam Y, Xiao D, Vignarajan J, Preetham A, Tay-Kearney ML, Mehrotra A: Evaluation of Artificial Intelligence-Based Grading of Diabetic Retinopathy in Primary Care. JAMA Netw Open2018, 1(5):e182665.

20. He J, Cao T, Xu F, Wang S, Tao H, Wu T, Sun L, Chen J: Artificial intelligence-based screening for diabetic retinopathy at community hospital. Eye (Lond)2020, 34(3):572-576.

21. Browning DJ, Stewart MW, Lee C: Diabetic macular edema: Evidence-based management. Indian J Ophthalmo/2018, 66(12):1736-1750.

22. Wang YT, Tadarati M, Wolfson Y, Bressler SB, Bressler NM: Comparison of Prevalence of Diabetic Macular Edema Based on Monocular Fundus Photography vs Optical Coherence Tomography. JAMA Ophthalmol2016, 134(2):222-228.

23. Kapoor R, Whigham BT, Al-Aswad LA: Artificial Intelligence and Optical Coherence Tomography Imaging. Asia Pac J Ophthalmol (Phila)2019, 8(2):187-194.

24. Graham PS, Kaidonis G, Abhary S, Gillies MC, Daniell M, Essex RW, Chang JH, Lake SR, Pal B, Jenkins AJet al: Genome-wide association studies for diabetic macular edema and proliferative diabetic retinopathy. BMC Med Genet2018, 19(1):71.

25. Wang FH, Liang YB, Zhang F, Wang JJ, Wei WB, Tao QS, Sun LP, Friedman DS, Wang NL, Wong TY: Prevalence of diabetic retinopathy in rural China: the Handan Eye Study. Ophthalmology2009, 116(3):461-467.

26. Wong TY, Cheung N, Tay WT, Wang JJ, Aung T, Saw SM, Lim SC, Tai ES, Mitchell P: Prevalence and risk factors for diabetic retinopathy: the Singapore Malay Eye Study. Ophthalmology2008, 115(11):1869-1875.

27. Wong TY, Klein R, Islam FM, Cotch MF, Folsom AR, Klein BE, Sharrett AR, Shea S: Diabetic retinopathy in a multi-ethnic cohort in the United States. Am J Ophthalmo/2006, 141(3):446-455.

28. Wong RL, Tsang CW, Wong DS, McGhee S, Lam CH, Lian J, Lee JW, Lai JS, Chong V, Wong IY: Are we making good use of our public resources? The false-positive rate of screening by fundus photography for diabetic macular oedema. Hong Kong Med J2017, 23(4):356-364.

29. Jyothi S, Elahi B, Srivastava A, Poole M, Nagi D, Sivaprasad S: Compliance with the quality standards of National Diabetic Retinopathy Screening Committee. Prim Care Diabetes2009, 3(2):67-72.

30. Kermany DS, Goldbaum M, Cai W, Valentim CCS, Liang H, Baxter SL, McKeown A, Yang G, Wu X, Yan Fet al: Identifying Medical Diagnoses and Treatable Diseases by Image-Based Deep Learning. Cel/2018, 172(5):1122-1131 e1129. 
31. Wang L, Wang G, Zhang M, Fan D, Liu X, Guo Y, Wang R, Lv B, Lv C, Wei Jet al: An Intelligent Optical Coherence Tomography-based System for Pathological Retinal Cases Identification and Urgent Referrals. Trans/ Vis Sci Techno/2020, 9(2):46.

32. Aldington SJ, Kohner EM, Meuer S, Klein R, Sjolie AK: Methodology for retinal photography and assessment of diabetic retinopathy: the EURODIAB IDDM complications study. Diabetologia1995, 38(4):437-444.

33. Abramoff MD, Folk JC, Han DP, Walker JD, Williams DF, Russell SR, Massin P, Cochener B, Gain P, Tang Let al: Automated analysis of retinal images for detection of referable diabetic retinopathy. JAMA Ophthalmol2013, 131(3):351-357.

34. Ren S, He K, Girshick R, Sun J: Faster R-CNN: Towards Real-Time Object Detection with Region Proposal Networks. IEEE Trans Pattern Anal Mach Intel/2017, 39(6):1137-1149.

35. Wu Z, Shi G, Chen Y, Shi F, Chen X, Coatrieux G, Yang J, Luo L, Li S: Coarse-to-fine classification for diabetic retinopathy grading using convolutional neural network. Artif Intell Med2020, 108:101936.

\section{Figures}

\section{Figure 1}

Examples of classification heatmaps of different levels of DR.

Figure 2

Retinal abnormality detection results. Detected retinal exudates (white bounding box) and retinal fluid (purple bounding box).

\section{Figure 3}

OCT-Fundus-Al diagnosis results. (a) OCT B-scan with detection of retinal fluid (purple bounding box). (b) OCT B-scan with detection of the epiretinal membrane (yellow bounding box). (c) No obvious abnormalities on the fundus. 


\section{Figure 4}

(a) Comparison of diabetic retinopathy (DR) grading between ophthalmologists and Al. (b) Comparison of macular oedema (ME) classifications between ophthalmologists and Al.

\section{Figure 5}

Venn diagram showing the overlap comparison of the number of referrals between human and automated grading: (a) fundus photography, (b) OCT, and (c) fundus photography combined with OCT. 